\title{
On identification of a flexible mechanical system using decimated data
}

\author{
Svante Gunnarsson \\ Division of Automatic Control \\ Department of Electrical Engineering \\ Linköpings universitet, SE-581 83 Linköping, Sweden \\ WWW: http://Www. control.isy.liu.se \\ E-mail: svante@isy.liu.se
}

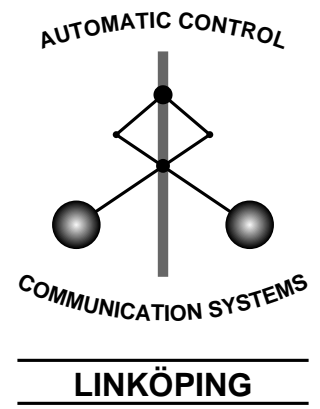

Report no.: LiTH-ISY-R-2653

Submitted to 16th IFAC World Congress

Technical reports from the Control \& Communication group in Linköping are available at http://www.control.isy.liu.se/publications. 


\begin{abstract}
System identification of a flexible mechanical system using decimated data is studied. It is illustrated how the use of decimated data can give erroneous results due to the inter-sample behavior of the signals, and an intuitive explanation to this phenomenon is proposed. The possible improvement by using alternative assumptions for the inter-sample behavior is investigated.
\end{abstract}

Keywords: Identification, physical models, continuous time systems, flexible arms, sampling 


\title{
ON IDENTIFICATION OF A FLEXIBLE MECHANICAL SYSTEM USING DECIMATED DATA
}

\author{
Svante Gunnarsson ${ }^{1}$ \\ Department of Electrical Engineering, Linköping University, \\ SE-581 83 Linköping, Sweden
}

\begin{abstract}
System identification of a flexible mechanical system using decimated data is studied. It is illustrated how the use of decimated data can give erroneous results due to the inter-sample behavior of the signals, and an intuitive explanation to this phenomenon is proposed. The possible improvement by using alternative assumptions for the inter-sample behavior is investigated.
\end{abstract}

Keywords: Identification, physical models, continuous time systems, flexible arms, sampling

\section{INTRODUCTION}

Identification of continuous time system using discrete time data has been been studied extensively by several authors. See (Unbehauen and Rao, 1998) for a thorough survey and e.g. (Rao and Garnier, 2002) for a recent contribution. Of particular practical interest are grey box models where the aim is to obtain estimates of the physical parameters in continuous time model structures using discrete time data. One important application area is motion control systems in general, see e.g. (Chou et al., 2003), and industrial robots, see e.g. (Rostgaard et al., 2001), (Daniel-Berhe and Unbehauen, 1997), in particular.

There are several possible approaches to this problem. One approach is to first estimate the frequency response of the system using some frequency domain method and then fit a parametric frequency response curve to the initial estimate. See e.g. (Suthasun et al., 2003). A second approach is to use a sequence of specially designed experiments where individual or subsets of the unknown parameters are estimated in each experiment. See e.g. (Isaksson et al., 2003). A third alternative, which is the one that will be applied here, is to estimate the parameters directly by using a time domain prediction error approach. See e.g. (Östring et al., 2003).

One important aspect of identification of continuous time systems using discrete time data is how to handle the inter-sample behavior of the data. In e.g. (Schoukens et al., 1994) it is shown how the violation of the assumed inter-sample behavior may lead to erroneous results. In some applications the frequency used during the data collection is determined by the hardware, and when the sampling frequency appears to be unnecessarily high it is appealing to decimate the input and output signals to a slower sampling rate. The purpose of this paper is to illustrate and explain some phenomena that can occur when system identification is carried out using decimated data.

The paper is organized as follows. In Section 2 the main ideas are illustrated using a first order example. Using these observations a two-mass flexible mechanical system is studied in Section 3. Finally some conclusions are given in Section 4 .

\footnotetext{
1 Supported by the Swedish Research Council.
} 


\section{FIRST ORDER SYSTEM}

\subsection{Problem description}

Consider a linear continuous time system with input $u(t)$, output $y(t)$, and transfer function $G(s)$. Assume that the input signal is applied to the continuous time system using zero order hold. The relationship between the input and output signals, in the sampling points, is given by the discrete time frequency response function $G_{T}\left(e^{i \omega T}\right)$. In general, for a given $\omega$ the frequency function will have larger negative phase shift for larger $T$, due to the delay caused by the hold function.

As an illustration consider a first order system with transfer function

$$
G(s)=\frac{100}{s+100}
$$

and assume that the input is a sinusoid with angular frequency $50 \mathrm{rad} / \mathrm{s}$. The input is generated using two different sampling intervals, $T_{1}=0.5$. $10^{-3} \mathrm{~s}$ and $T_{2}=5 \cdot 10^{-3} \mathrm{~s}$ respectively. Figure 2.1 shows the frequency functions $G_{T_{1}}\left(e^{i \omega T_{1}}\right)$ and $G_{T_{2}}\left(e^{i \omega T_{2}}\right)$ respectively. At $\omega=50 \mathrm{rad} / \mathrm{s}$ the phase difference between the two frequency functions is approximately $7^{\circ}$.

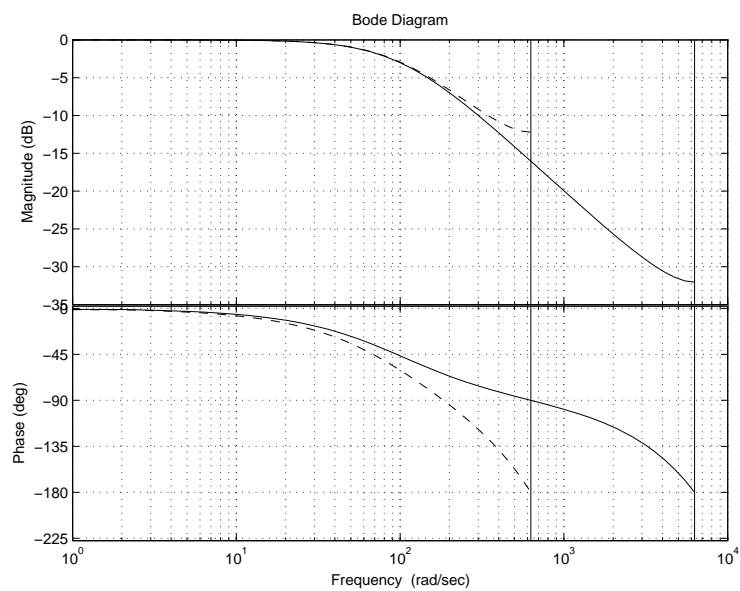

Fig. 1. Discrete time frequency function of the system given by equation (1). Solid: $T=0.5$. $10^{-3}$. Dashed: $T=5 \cdot 10^{-3}$.

The continuous time system, equation (1), is simulated using zero-order hold input and the sampling intervals $T_{1}$ and $T_{2}$ respectively. Figures 2 and 3 show the input and output signals, and the difference in phase shift is clearly seen in Figure 3 .

\subsection{Black-box identification}

Assume now that the signals from the simulation using sampling interval $T_{1}$ (solid lines in Figures 2 and 3 ) are decimated by a factor 10 . Since there

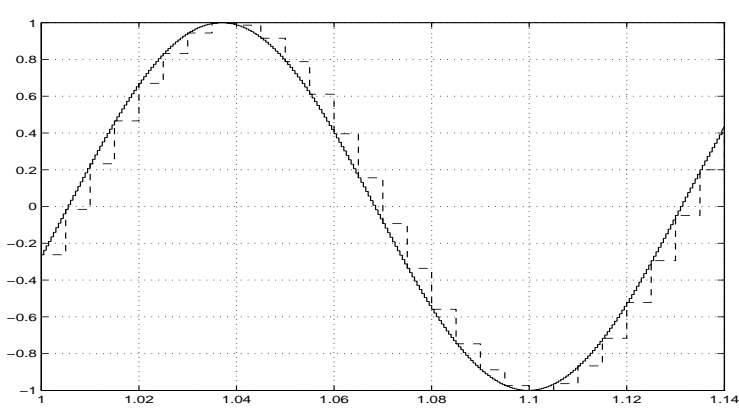

Fig. 2. Input signal. Solid: $T=0.5 \cdot 10^{-3}$. Dashed: $T=5 \cdot 10^{-3}$

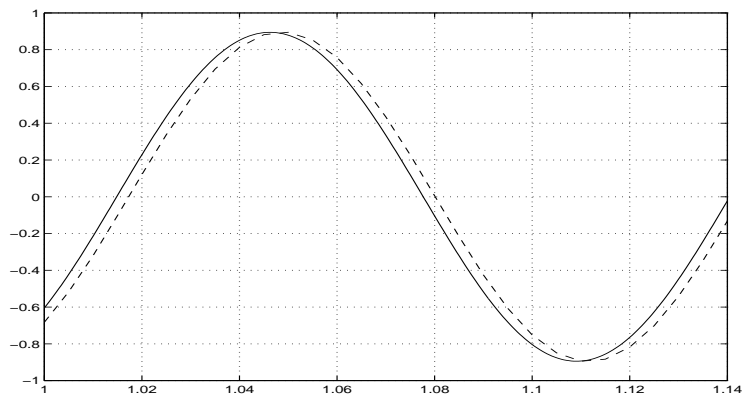

Fig. 3. Output signal. Solid: $T=0.5 \cdot 10^{-3}$. Dashed: $T=5 \cdot 10^{-3}$

are no disturbances present the decimation can be done by simply picking every tenth sample of the input and output vectors. Using the decimated signals in discrete time black box identification implies that zero order hold input is assumed, and for the input this corresponds to the dashed curve in figure 2. This input signal is combined with the decimated version of the solid curve in 3 , and the resulting data set is used for identification. For sampling interval $T_{2}$ the true discrete time transfer function $G_{T_{2}}$ describes the relationship between the dashed input in Figure 2 and dashed output signal in Figure 3. The system identification, on the other hand, tries to find a model that describes the relationship between the dashed input in Figure 2 and the solid output in Figure 3. Due to the phase difference between the solid and dashed output signals the true input/output relationship will not belong to the model class, and the resulting model will be biased. The model fit can be characterized by the bias integral presented in e.g.(Ljung, 1999). Assume that the true relationship between the input and output signal is given by the transfer operator $G_{0}(q)$ and the model structure is given by $G(q, \theta)$. The asymptotic model, as the number of data tends to infinity, is given by

$$
\begin{aligned}
\theta^{*} & =\arg \min _{\theta} \int_{-\pi / T}^{\pi / T}\left|G_{0}\left(e^{i \omega T}\right)-G\left(e^{i \omega T}, \theta\right)\right|^{2} \\
& \times \Phi_{u}(\omega) d \omega
\end{aligned}
$$

The model fit will depend on the properties of the excitation signal used for identification. Here 
the input is a a single frequency sinusoid and it implies that the identified model will have correct amplification and phase shift at the frequency of the sinusoid. A first order output error (OE) model is identified using the decimated data set. Transforming back the estimated model to continuous time results in the model

$$
\hat{G}_{z o h}(s)=\frac{135}{s+143}
$$

i.e. a substantial error in both time constant and static gain. The frequency functions of the true and estimated models are shown in Figure 2.2. The model tries to match the negative phase shift between the dashed input and the solid output, which is less than for the true transfer function for sampling interval $T_{2}$. It is therefore natural that the pole of the model is moved to higher frequency in order to match the phase shift.

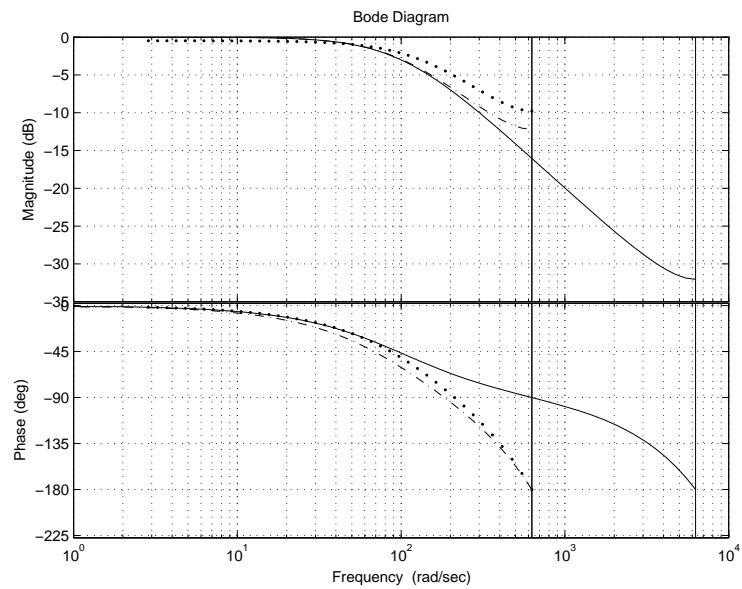

Fig. 4. Discrete time frequency functions. Solid: True system for $T=0.5 \cdot 10^{-3}$. Dashed: True system for $T=5 \cdot 10^{-3}$. Dashed-dotted: Estimated model

\subsection{Grey-box identification}

An alternative to the approach used above above is to use the idgrey model structure in the System Identification Tool-box, see (Ljung, 2000). The continuous time model is defined as a state space model

$$
\begin{aligned}
& \dot{x}(t)=A(\theta) x(t)+B u(t)+K e(t) \\
& y(t)=C(\theta) x(t)+e(t)
\end{aligned}
$$

and the matrices $A(\theta), B(\theta), C(\theta)$, and $K(\theta)$ are specified in an $\mathrm{m}$-file. The user of the tool-box can, by using the appropriate options, control how the inter-sample properties, specified in the data object, will affect the identification. By specifying that the m-file always delivers the continuous time system matrices the inter-sample property determines if zero order hold or first order hold is used when computing the discrete time predictor.
For the first order example this gives that zeroorder hold inter-sample behavior of the decimated data set yields the same model as in (3). First order hold character of the input will give a better, but of course not perfect, description of the input character. In this case the estimated continuous time model is given by

$$
\hat{G}_{f o h}(s)=\frac{98}{s+97}
$$

Figure 2.3 shows that the estimated continuous time model agrees very well with the true continuous time system.

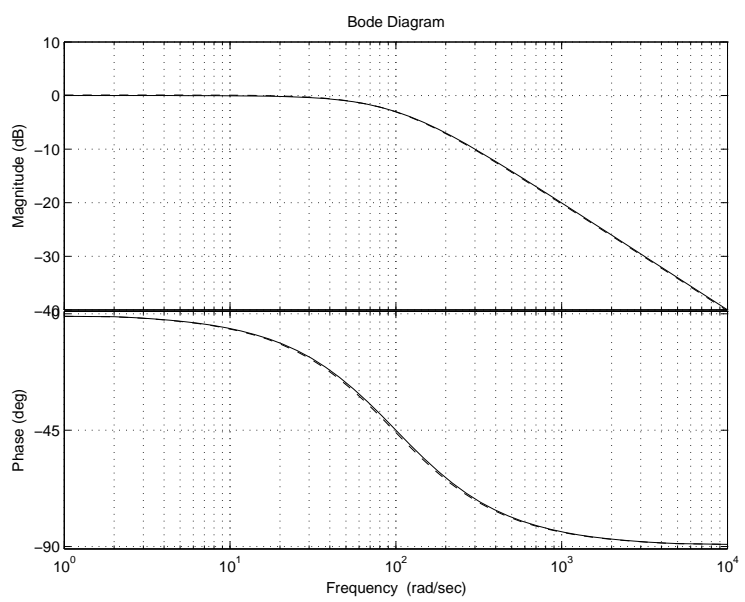

Fig. 5. Continuous time frequency functions. Solid: True system. Dashed: Estimated model uing $T=5 \cdot 10^{-3}$ and first order hold input.

\subsection{Conclusion}

The example illustrates that the assumption that the decimated data set has zero order hold intersample properties can give erroneous result due the phase difference of the output at different sampling rates. It also shows that the error will depend on the frequency contents of the input signal. Considerably better results are obtained by identifying the continuous time model directly and specifying first order hold input character of the decimated data set.

\section{TWO-MASS MECHANICAL SYSTEM}

\subsection{Problem description}

Consider now a two-mass flexible mechanical system shown in Figure 3.1

Here $J_{m}$ and $J_{a}$ denote the moments of inertia of the first and second mass respectively. The parameters $k$ and $d$ denote the stiffness and damping of the spring respectively, and $f_{m}$ and $r$ denote the viscous friction of the first mass and the gear ratio 


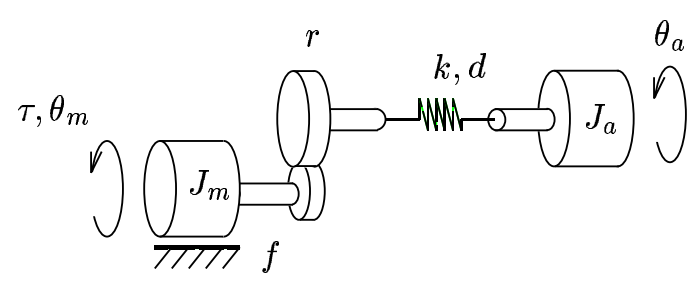

Fig. 6. Two-mass model

respectively. Torque balances of the two masses give

$$
\begin{aligned}
J_{m} \ddot{\theta}_{m}(t) & =-f \dot{\theta}_{m}(t)-r k\left(r \theta_{m}(t)-\theta_{a}(t)\right) \\
& -r d\left(r \dot{\theta}_{m}(t)-\dot{\theta}_{a}(t)\right)+k_{\tau} u(t)
\end{aligned}
$$

and

$$
\begin{aligned}
J_{a} \ddot{\theta}_{a}(t) & =k\left(r \theta_{m}(t)-\theta_{a}(t)\right) \\
& +d\left(r \dot{\theta}_{m}(t)-\dot{\theta}_{a}(t)\right)
\end{aligned}
$$

respectively. Considering the torque as input signal, the angular velocity of the first mass as output signal, and using the state variables $x_{1}(t)=$ $r \theta_{m}(t)-\theta_{a}(t), x_{2}(t)=\dot{\theta}_{m}, x_{3}(t)=\dot{\theta}_{a}(t)$ the equations (7) and (8) give

$$
\dot{x}(t)=A x(t)+B u(t) \quad y(t)=C x(t)
$$

where

$$
\begin{gathered}
A=\left(\begin{array}{ccc}
0 & r & 1 \\
-\frac{r k}{J_{m}} & -\frac{f+r^{2} d}{J_{m}} & \frac{r d}{J_{m}} \\
\frac{k}{J_{a}} & \frac{d r}{J_{a}} & -\frac{d}{J_{a}}
\end{array}\right) \\
B=\left(\begin{array}{lll}
0 & \frac{1}{J_{m}} & 0
\end{array}\right)^{T} \\
C=\left(\begin{array}{lll}
0 & 1 & 0
\end{array}\right)
\end{gathered}
$$

In the sequel the parameter values given in Table 1 will be used. The gear ratio $r=1 / 118$ is known a priori.

Table 1. Nominal parameter values

\begin{tabular}{ll} 
Parameter & Nominal value \\
\hline$J_{a}$ & 11 \\
$k$ & $1.5 \cdot 10^{5}$ \\
$J_{m}$ & $9 \cdot 10^{-4}$ \\
$f_{m}$ & $1 \cdot 10^{-3}$ \\
$d$ & 10 \\
\hline
\end{tabular}

The model defined by equation (12) and Table 1 represents a realistic description of the dynamics of an ABB IRB 1400 industrial robot when moving around axis one. See e.g. (Östring et al., 2001)

The transfer function of the system is given by

$$
G(s)=\frac{B(s)}{A(s)}
$$

where

$$
\begin{aligned}
B(s) & =J_{a} s^{2}+d s+k \\
A(s) & =J_{a} J_{m} s^{3}+s^{2}\left(J_{a} f_{m}+d\left(J_{m}+r^{2} J_{a}\right)\right) \\
& +s\left(k\left(J_{m}+r^{2} J_{a}\right)+d f_{m}\right)+k f_{m}
\end{aligned}
$$

Assuming zero order hold the corresponding discrete time transfer functions are computed, and the corresponding frequency functions are shown in Figure 7. The figure shows that the discrete time frequency function corresponding to $T=5$. $10^{-3}$ has larger negative phase shift.

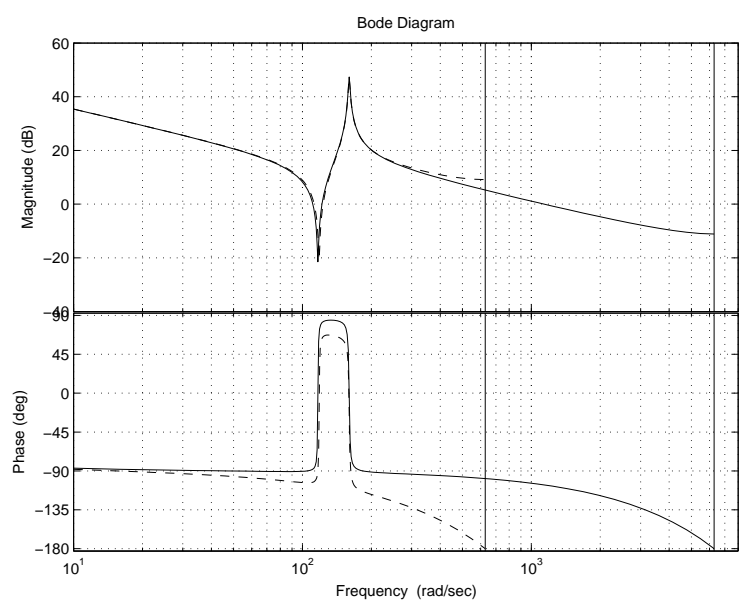

Fig. 7. Discrete time frequency function of the system given by equation (14). Solid: $T=0.5$. $10^{-3}$. Dashed: $T=5 \cdot 10^{-3}$.

\subsection{Black-box identification}

The input is chosen as a chirp signal, i.e. a sinusoid where the frequency changes from 10 to $80 \mathrm{~Hz}$ during the experiment. The experiment lasts for 10 seconds, and the sampling interval is, like in the first order case, $0.5 \cdot 10^{-3}$ seconds. The continuous time system is simulated using zero order hold input, and the input and output signals are decimated by a factor ten before the identification is carried out. A third order output error model is identified, and the resulting model is shown in Figure 8. It is clearly seen that the identified model differs a substantially from true discrete time system. The notch frequency is essentially lower for the identified model and the phase curve has a different behavior.

The properties of the identified model are also revealed by studying the Nyquist curves of the frequency functions as shown in Figure 9. The figure shows that the Nyquist curve of the estimated model, with sampling interval $T_{2}$, follows the Nyquist curve of the true frequency function corresponding the $T_{1}$, i.e. the shorter sampling interval. This is in agreement with the observations from the first order example. The identified model tries to model, within the frequency range of the input, the relationship between the input signal 

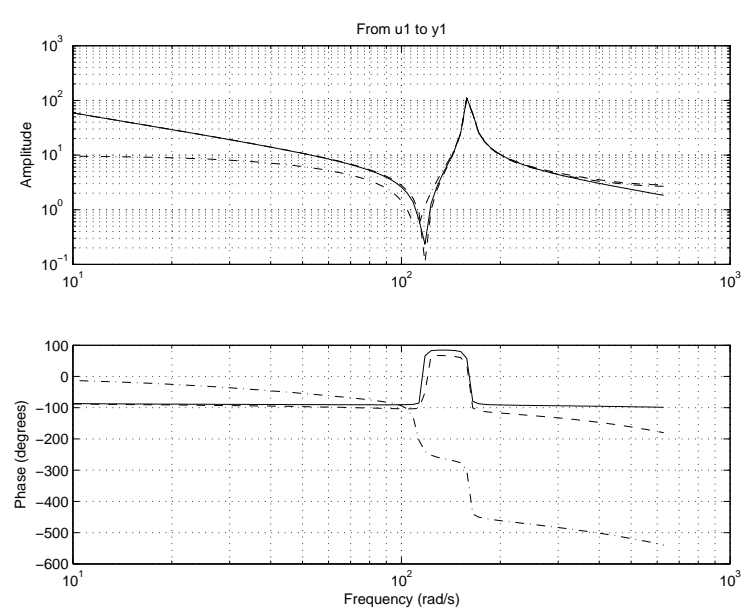

Fig. 8. Discrete time frequency functions. Solid: True system for $T=0.5 \cdot 10^{-3}$. Dashed: True system for $T=5 \cdot 10^{-3}$. Dashed-dotted: Estimated model

and the output signal corresponding to the shorter sampling interval. See also the bias integral (2). It has to be noted that the achieved model depends on where the energy of the input is located.

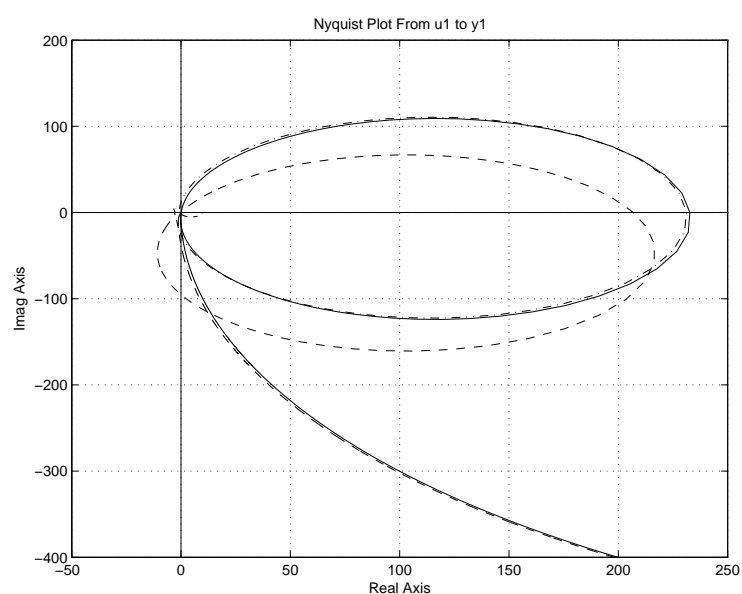

Fig. 9. Discrete time frequency functions. Solid: True system for $T=0.5 \cdot 10^{-3}$. Dashed: True system for $T=5 \cdot 10^{-3}$. Dashed-dotted: Estimated model

An important property of the identified model is that the zeros of the model are located outside the unit circle, and when the discrete time model is converted to continuous time the zeros are located in the right half plane. The estimated numerator polynomial is given by

$$
N(s)=1.0 \cdot 10^{3} s^{2}-5.1 \cdot 10^{3} s+1.3 \cdot 10^{6}
$$

A comparison of this polynomial and the numerator of equation (14) shows that the estimated damping $d$ is negative, which is a non-physical results.

\subsection{Grey-box identification}

The identification of the two-mass mechanical model can be carried out in a similar way as for the first order example above. The starting point is an $\mathrm{m}$-file defining the structure of the physically parameterized model. This structure follows from the state space model given by (12). In order to improve the behavior of the identification procedure it has been found to useful to scale the physical parameters such that they all are of the same order of magnitude. A second action is to view the inverses of the moments of inertia as unknown parameters rather than the physical parameters themselves. This has also turned out to affect the results positively.

The system is simulated for 10 seconds, using zero order hold input and sampling interval $T=0.5$. $10^{-3}$. The input and output data vectors are decimated by a factor 10 . Using the decimated data set two sets are defined. In the first set the inter-sample behavior is set to be zero order hold and in the second set it is set to be first order hold. The results from identifications using these sets are presented in Table 2 and Figure 10. The estimated frequency function assuming first order hold input gives a considerably better result. The estimated damping is positive and the other parameters are fairly close to their nominal values.
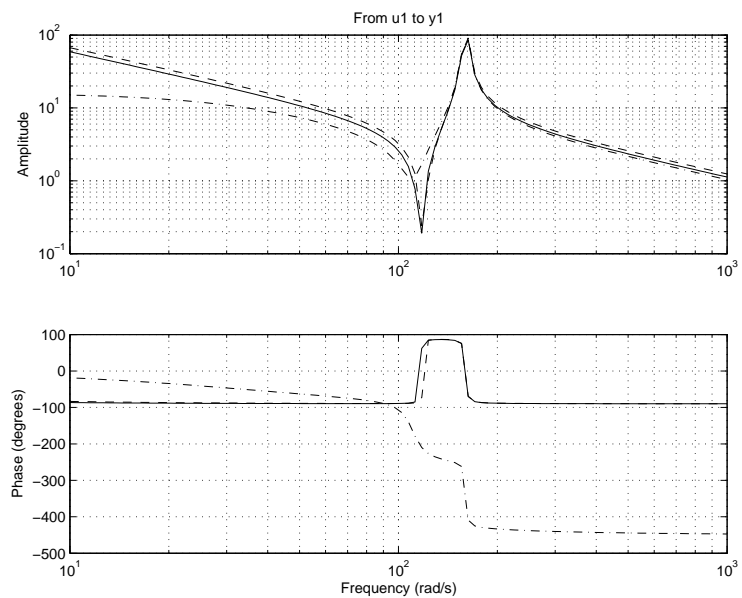

Fig. 10. Continuous time frequency functions. Solid: True system. Dashed: Estimated model assuming first order hold input. Dash-dotted: Estimated model assuming zero order hold input.

Table 2. Nominal parameter values and estimated parameter values for zero and first order hold respectively

\begin{tabular}{llll}
\hline Par. & Nom. value & $\mathrm{ZOH}$ & $\mathrm{FOH}$ \\
\hline$J_{m}$ & $9 \cdot 10^{-4}$ & $11 \cdot 10^{-4}$ & $8.2 \cdot 10^{-4}$ \\
$k$ & $1.5 \cdot 10^{5}$ & $1.9 \cdot 10^{5}$ & $1.3 \cdot 10^{5}$ \\
$J_{a}$ & 11 & 16 & 9.3 \\
$f_{m}$ & $1 \cdot 10^{-3}$ & $63 \cdot 10^{-3}$ & $1.7 \cdot 10^{-3}$ \\
$d$ & 10 & -230 & 6.5 \\
\hline
\end{tabular}


Figure 11 shows once more the phenomenon noted above. The estimated model, assuming zero order hold input, for the longer sampling interval tries to match the true frequency function corresponding to the shorter frequency interval within the frequency range of the input.

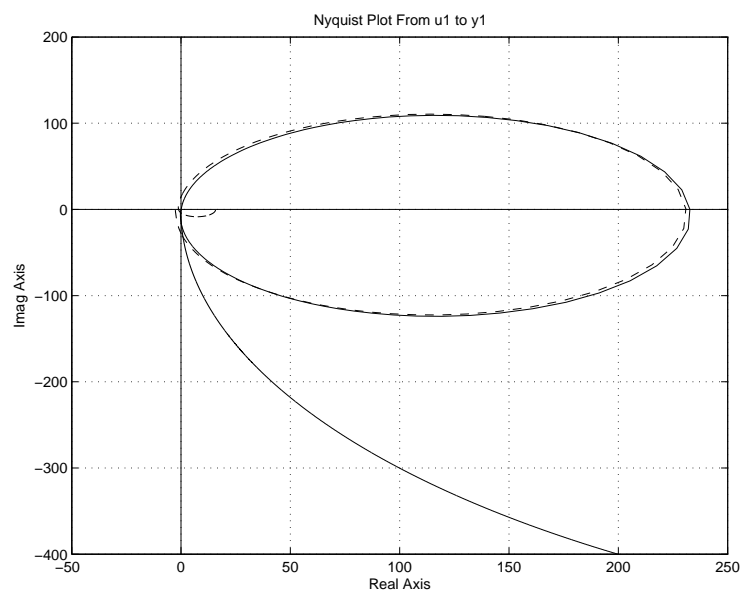

Fig. 11. Discrete time frequency functions. Solid: True system for $T=0.5 \cdot 10^{-3}$. Dashed: Estimated model using zero order hold input converted to discrete time using $T=5 \cdot 10^{-3}$

\section{CONCLUSIONS}

The consequences of using decimated data for identification of continuous time systems have been investigated. It has been illustrated that decimation of the data may lead to erroneous models and in some cases models without physical interpretation. The errors are caused by the violation of the assumption that the input is piecewise constant during the sampling interval. A possible interpretation of the behavior is that the error is caused by the difference in phase shift of the output signal for different sampling intervals. One way to improve the results is to identify the continuous time model directly and assume first order hold input of the input signal. The results are illustrated by identification of the physical parameters of a two-mass flexible mechanical system.

\section{REFERENCES}

Chou, J.H., J.H. Sun and J.N. Shieh (2003). On-line identification and optimal control of continuous-time systems. Mathematics and Computers in Simulation 63, 493-503.

Daniel-Berhe, S. and H. Unbehauen (1997). Physical parameter estimation of the nonlinear dynamics of a single link robotic manipulator with flexible joint using the hmf method. In: Proc. of the 1997 American Control Conference. Albuquerque, New Mexico. pp. 15041508.
Isaksson, A.J., R. Lindkvist, X. Zhang, M. Nordin and M. Tallfors (2003). Identification of mechanical parameters in drive train systems. In: 13th IFAC Symposium on System Identification. Rotterdam, Netherlands.

Ljung, L. (1999). System Identification: Theory for the User. 2:nd ed.. Prentice-Hall. Upper Saddle River, N.J. USA.

Ljung, L. (2000). System Identification ToolboxUser's Guide. The MathWorks Inc. Sherborn, MA, USA.

Rao, G.P. and H. Garnier (2002). Numerical illustrations of the relevance of direct continuoustime model identification. 15th IFAC World Congress.

Rostgaard, M., N.K. Poulsen and O. Ravn (2001). Pay-load estimation of a 2dof flexible link using a delta-operator technique. In: Proc. of the 2001 IEEE International Conference on Control Applications. Mexico City, Mexico. pp. 248-253.

Schoukens, J., R. Pintelon and H. van Hamme (1994). Identification of linear dynamic systems using piecewise constant excitations: Use, misuse and alternatives. Automatica 30, 1153-1169.

Östring, M., S. Gunnarsson and M. Norrlöf (2001). Closed loop identification of physical parameters of an industrial robot. In: 32nd International Symposium on Robotics. Seoul, Korea.

Östring, M., S. Gunnarsson and M. Norrlöf (2003). Closed loop identification of an industrial robot containing flexibilitie. Control Engineering Practice 11, 291-300.

Suthasun, T., I. Mareels and A. Al-Mamun (2003). System identification and controller design for dual actuated hard disk drive. Control Engieneering Practice 12, 665-676.

Unbehauen, H. and G. P. Rao (1998). A review of identification of continuous-time systems. Annual Reviews in Control 22, 145-171. 